Enfance en difficulté

\title{
Caractéristiques individuelles et familiales associées à la persistance des troubles du comportement perturbateur chez les enfants
}

\author{
Melissa Goodfellow, Mélanie Lapalme, Michèle Déry et Annie Lemieux
}

Volume 5, novembre 2017

URI : https://id.erudit.org/iderudit/1043353ar

DOI : https://doi.org/10.7202/1043353ar

Aller au sommaire du numéro

Éditeur(s)

Université Laurentienne

ISSN

1920-6275 (imprimé)

1929-8544 (numérique)

Découvrir la revue

Citer cet article

Goodfellow, M., Lapalme, M., Déry, M. \& Lemieux, A. (2017). Caractéristiques individuelles et familiales associées à la persistance des troubles du comportement perturbateur chez les enfants. Enfance en difficulté, 5, 25-54. https://doi.org/10.7202/1043353ar
Résumé de l'article

Caractéristiques individuelles et familiales associées à la persistance des troubles du comportement perturbateur chez les enfants. Cette étude vise à identifier des caractéristiques distinguant les enfants des trajectoires persistante et décroissante des troubles de comportement perturbateur et de vérifier l'effet modérateur du genre. L'échantillon est composé de 268 enfants ( $28 \%$ de filles) du primaire ( $M=9,8$ ans; é-t. $=1,79$ an) recevant des services spécialisés pour difficultés comportementales à l'école. Selon les analyses de trajectoires (six ans) réalisées, 12,3\% d'enfants ( $24 \%$ de filles) appartiennent à la trajectoire persistante et 32,5\% (30\% de filles) à la trajectoire décroissante. Bien que les enfants des deux trajectoires présentent des vulnérabilités similaires à l'entrée dans l'étude, les analyses de régression multivariées indiquent qu'un tempérament plus réactif augmente la probabilité d'appartenir à la trajectoire persistante chez les filles. Aussi, la présence d'un trouble anxieux et l'absence d'un trouble dépressif chez les parents seraient associées à la trajectoire persistante. Ces résultats soutiennent l'importance de cibler aussi les difficultés des parents lorsque l'on souhaite prévenir la persistance des troubles du comportement.
Ce document est protégé par la loi sur le droit d'auteur. L'utilisation des services d'Érudit (y compris la reproduction) est assujettie à sa politique d'utilisation que vous pouvez consulter en ligne.

https://apropos.erudit.org/fr/usagers/politique-dutilisation/ 
www.laurentienne.ca

\title{
Caractéristiques individuelles et familiales associées à la persistance des troubles du comportement perturbateur chez les enfants
}

\author{
Melissa Goodfellow, Mélanie Lapalme, Michèle Déry et \\ Annie Lemieux \\ Université de Sherbrooke
}

\section{Introduction}

Les troubles de comportement perturbateur (TCP), dont le trouble oppositionnel avec provocation et le trouble des conduites, sont parmi les troubles mentaux les plus fréquemment rencontrés chez les enfants (American Psychiatric Association; APA, 2013). Ces troubles se caractérisent par la manifestation de conduites antisociales telles que la confrontation avec les figures d'autorité et la violation des règles et des droits d'autrui, entrainant des difficultés d'adaptation importantes chez les jeunes et pouvant perturber largement leur environnement social (APA, 2013). Les TCP sont généralement plus communs chez les garçons que chez les filles (APA, 2013; Meltzer, Gatwood, Goodman et Ford, 2000), mais cela peut varier selon le trouble ou l'âge des enfants (APA, 2013). Par exemple, la prévalence du trouble oppositionnel avec provocation varie de $1 \%$ à $11 \%$ (Canino, Polanczyk, Bauermeister, Rohde et Frick, 2010). À l'enfance, on le retrouve davantage chez les garçons que chez les filles (1,4:1), mais cet écart s'estompe à l'adolescence (Boylan, Vaillancourt, Boyle et Szatmari, 2007). En ce qui a trait au trouble des conduites, les taux de prévalence varient de $2 \%$ à plus de $10 \%$ (Costello, Egger et Angold, 2005); ils sont généralement plus élevés 
à l'adolescence et deux fois plus élevés chez les garçons que chez les filles (Lussier et Flessas, 2009).

Bien que les TCP soient plus fréquents à l'adolescence, plus ils surviennent tôt à l'enfance, plus le pronostic est sombre. En effet, l'apparition de ces troubles à l'enfance est associée à plus de problèmes d'adaptation avec les pairs et à l'école (Déry, Toupin, Pauzé et Verlaan, 2005) et ces jeunes seraient plus enclins à la consommation précoce de psychotropes (Biederman et al., 2008; Rowe, Costello, Angold, Copeland et Maughan, 2010). À l'âge adulte, plusieurs éprouveront des difficultés d'adaptation dans leur vie familiale et sur le marché du travail (Beekhoven et Dekkers, 2005) et adopteront des comportements criminels (Fergusson, Boden et Horwood, 2009; Moffitt, Caspi, Dickson, Silva et Stanton, 1996; Moffitt, Caspi, Harrington et Milne, 2002; Piquero, Farrington, Nagin et Moffitt, 2010, Rowe et al., 2010). Des études soulignent que les conséquences seraient encore plus sévères chez les filles que chez les garçons, notamment en termes de troubles intériorisés (Loeber et Keenan, 1994; Zahn-Waxler et Polanichka, 2004), de parentalité précoce et de transmission intergénérationnelle (Serbin et al., 1998). Il importe donc de les considérer distinctement des garçons.

Si les troubles qui surviennent en bas âge sont reconnus pour leur risque élevé de persistance dans le temps (Biederman et al., 2008; Lahey, Loeber, Burke et Applegate, 2005; Renk, 2008; Whittinger, Langley, Fowler, Thomas et Thapar, 2007) chez un nombre important d'enfants, ceux-ci décroitront (Barker et Maughan, 2009; Hoeve et al., 2008; Moffitt et al. 1996; Pitzer, Schmidt et Laucht, 2010; Raine et al., 2005; Van Domburgh, Loeber, Bezemer, Stallings et Stouthamer-Loeber, 2009; Veenstra, Lindenberg, Verhulst et Ormel, 2009). En effet, environ la moitié des jeunes ayant manifesté de telles conduites tôt à l'enfance n'en présentent plus un niveau élevé à l'adolescence (Côté, Vaillancourt, LeBlanc, Nagin et Tremblay, 2006; Moffitt et al., 1996). Lidentification de vulnérabilités à l'enfance qui distingueraient les enfants chez qui ces troubles persistent de ceux dont les troubles décroissent s'avère donc une avenue prometteuse afin de prioriser et d'ajuster les contextes et cibles d'interventions susceptibles de prévenir la persistance des TCP.

Les différents modèles explicatifs du développement et de la persistance des TCP (Lahey, Waldman et McBurnett, 1999; Lahey et Waldman, 2003; Moffitt, 1993; Moffitt, 2006; Moffitt et al., 1996) qui ont guidé le choix des variables à l'étude font habituellement état de deux grandes catégories, soit les caractéristiques propres à l'enfant et celles de 
son environnement. L’apparition en bas âge de ces troubles, leur sévérité et leur persistance dans le temps s'expliqueraient par l'interaction entre des déficits neuropsychologiques (les fonctions verbales et exécutives, un tempérament difficile et des difficultés de socialisation) et les vulnérabilités propres aux parents (un environnement criminogène et des pratiques éducatives inadéquates). Donc, en raison de leurs déficits neuropsychologiques, ces enfants opposeraient plus de résistance au contrôle exercé par leurs parents qui, eux, seraient peu outillés pour répondre adéquatement aux besoins plus grands de leur enfant et pour gérer leurs comportements difficiles (Moffitt, 1993). Ces vulnérabilités pourraient être liées à la précocité, à la sévérité et à la persistance des TCP. De ces modèles, on pourrait poser l'hypothèse que parmi les enfants présentant des TCP tôt à l'enfance, il y aurait décroissance chez ceux présentant moins de vulnérabilités ou des vulnérabilités individuelles et familiales moins sévères.

Si les vulnérabilités explicitement associées à la présence de TCP chez les enfants sont nombreuses (Moffitt, 1993), celles qui distinguent les enfants dont les TCP persistent de ceux dont les TCP se résorbent sont moins bien documentées. Bien que plusieurs études aient été réalisées sur cette question et que certaines donnent un appui aux modèles explicatifs du développement et de la persistance de ces troubles (Barker et Maughan, 2009; Hoeve et al., 2008; Moffitt et al. 1996; Pitzer et al., 2010; Raine et al., 2005; Van Domburgh et al., 2009; Veenstra et al., 2009), les résultats sont peu concluants. D'une part, certaines études où l'on a comparé ces vulnérabilités entre les enfants dont les troubles ont persisté et ceux dont les troubles ont décru, soutiennent que ceux dont les troubles ont persisté auraient un tempérament plus difficile (Barker et Maughan, 2009; Veenstra et al., 2009) et entretiendraient des relations avec les pairs moins harmonieuses (Van Domburgh et al., 2009). D'autre part, pour ce qui est des vulnérabilités familiales, les parents de ces enfants auraient une santé mentale plus précaire (Barker et Maughan, 2009), présenteraient plus de comportements antisociaux (Veenstra et al., 2009) et adopteraient des pratiques éducatives moins adéquates (Barker et Maughan, 2009; Veenstra et al., 2009).

Malgré ces constats, les résultats divergent souvent selon les études. En effet, les caractéristiques individuelles et familiales seraient tantôt moins favorables, tantôt similaires, voire moins problématiques chez les enfants dont les troubles ont persisté (Barker et Maughan, 2009; Pitzer et al., 2010; Raine et al., 2005; Van Domburgh et al., 2009; Veenstra et al., 2009). Quant à savoir si les résultats s'appliquent autant aux 
garçons qu'aux filles, seulement deux études ont porté une attention particulière aux différences entre les genres en étudiant les garçons et les filles séparément (Barker et Maughan, 2009; Pitzer et al., 2010). Ainsi, les filles de la trajectoire persistante comparées à celle de la trajectoire décroissante présenteraient des difficultés qu'on ne retrouve pas chez les garçons telles que plus de problèmes d'attention (Pitzer et al., 2010), davantage d'adversité familiale (Barker et Maughan, 2009; Pitzer et al., 2010) et leur mère bénéficierait d'un moins grand soutien social (Barker et Maughan, 2009). Ces résultats demeurent bien évidemment à explorer davantage tout en considérant les vulnérabilités des garçons et des filles, séparément.

Bien que les caractéristiques associées aux différentes trajectoires des TCP soient bien documentées, les caractéristiques qui permettent de distinguer les trajectoires persistante et décroissante ne ressortent pas clairement. De plus, les études ayant abordé la question démontrent des faiblesses méthodologiques ayant pu nuire à une meilleure compréhension du phénomène. Par exemple, aucune d'entre elles n'a tenu compte des services reçus. Il est pourtant fort probable qu'une proportion significative des enfants composant les échantillons étudiés ait reçu des services pour leurs difficultés comportementales, notamment dans les études portant sur un échantillon clinique (Hoeve et al., 2008; Pitzer et al., 2010; Raine et al., 2005; Van Domburgh et al., 2009). Par conséquent, il est difficile de déterminer si la décroissance des TCP peut s'expliquer par la fréquence des services reçus plutôt que par la présence de vulnérabilités moindres ou de vulnérabilités individuelles et familiales moins problématiques.

Aussi, certaines études ne contrôlent pas l'équivalence des TCP présentés au départ, et ce, entre les trajectoires persistante et décroissante (Barker et Maughan, 2009; Pitzer et al., 2010; Van Domburgh et al., 2009). Comme la persistance est reconnue pour son association à une plus grande sévérité des TCP initiaux (Barker et Maughan, 2009; Pitzer et al., 2010; Van Domburgh et al., 2009), ces études ne permettent pas de vérifier si la décroissance des troubles s'explique par des vulnérabilités moindres ou par la présence de difficultés comportementales moins sévères au départ.

\section{Objectifs de l'étude}

La présente recherche vise à apporter des précisions sur les différences entre les trajectoires persistantes et décroissantes en tentant de 
combler les lacunes des études recensées. D'abord, différentes trajectoires des TCP sont établies sur une période de six ans (4 temps de mesure) à partir d'un échantillon d'enfants ayant des difficultés comportementales à l'école primaire. Ensuite, l'étude permet de vérifier si des caractéristiques individuelles et familiales mesurées à l'entrée dans l'étude (au temps 1) contribuent à différencier les enfants appartenant aux trajectoires persistantes et décroissantes et de vérifier l'effet modérateur du genre sur ces caractéristiques. Le choix des caractéristiques évaluées repose sur les modèles théoriques ainsi que sur les caractéristiques individuelles (fonctions verbales et exécutives, tempérament et intégration sociale) et familiales (pratiques éducatives, criminalité dans la famille et santé mentale des parents) émergeant des études recensées. Rappelons l'hypothèse à l'effet que les enfants de la trajectoire décroissante seraient confrontés à moins de vulnérabilités que les enfants de la trajectoire persistante. Léquivalence de la sévérité des TCP présentés par les enfants à l'entrée dans l'étude a été contrôlée entre les trajectoires, de même que la fréquence des services reçus. Les connaissances issues de cette recherche permettront de sensibiliser les intervenants et de les aider à identifier des cibles d'intervention prioritaire afin de prévenir la persistance des TCP.

\section{Méthodologie}

\section{Échantillon}

Cette étude porte sur un échantillon composé de garçons et de filles âgés de 6 à 13 ans $^{1}$ au premier temps de mesure issu de l'étude longitudinale (1999-2010) portant sur la persistance des troubles de comportement (voir Déry, Toupin, Pauzé et Verlaan, 2004). L'échantillon initial $(n=324)$ a été recruté en 1999, 2000 et 2001, dans des écoles primaires de trois commissions scolaires situées en Estrie et en Montérégie. En 2004, une cohorte supplémentaire strictement composée de filles ( $n=38$ ) provenant de trois autres commissions scolaires de l'Estrie et de la Montérégie a été ajoutée à l'échantillon afin d'avoir un nombre suffisant de filles pour étudier spécifiquement leur profil comportemental. Ces filles sont comparables aux filles des autres cohortes, tant sur le plan de l'âge et de la sévérité que des TCP présentés. Léchantillon total est donc composé de 362 enfants ( $28 \%$ de filles). Il se veut représentatif de la population

$90 \%$ des enfants retenus dans la présente étude ont moins de 12 ans et seulement 4 enfants viennent tout juste d'avoir 13 ans. Ces enfants ont été conservés dans l'échantillon puisqu'ils fréquentaient tous l'école primaire. 
cible sur le plan de la provenance des enfants, de la répartition dans chaque niveau scolaire et du taux de scolarisation en classe spéciale. Les filles sont légèrement surreprésentées dans l'échantillon en raison de l'ajout de la quatrième cohorte. Chaque cohorte a été évaluée au premier temps de mesure, puis à tous les deux ans, pendant 6 ans.

Pour la présente étude, seuls les participants pour lesquels les données étaient disponibles à au moins deux temps de mesure — nécessairement au temps 1 , puis à au moins un temps subséquent - ont été retenus, soit 268 enfants. Parmi ceux-ci, il y a 192 garçons et 76 filles âgés de 9,8 ans en moyenne (é.-t. = 1,79 an). Les 94 enfants exclus ne se différencient pas significativement des enfants inclus quant au nombre de symptômes de TCP au premier temps de mesure, $t(156,36)=-0,455 ; p=0,649$.

\section{Déroulement}

Les parents des enfants ont d'abord été contactés par les intervenants du milieu scolaire pour les informer du programme de recherche et demander s'ils voulaient y participer. Ces intervenants ont ensuite transmis au personnel de recherche les coordonnées des parents qui avaient donné une réponse positive. Les enfants et leur parent, celui qui s'occupe le plus de prodiguer les soins à son enfant, ont été rencontrés individuellement à domicile par des intervieweurs préalablement formés à la passation des instruments de mesures. Au début de la rencontre, le parent signait un formulaire de consentement pour participer à l'étude et donnait son autorisation pour que le personnel de recherche communique avec l'enseignant de leur enfant qui, lui, donnait son consentement verbal. L'enseignant de chaque enfant était ensuite contacté par téléphone pour effectuer la passation des instruments de mesures. Ces mêmes entrevues diagnostiques ont été effectuées au deuxième, au troisième et au quatrième temps de mesure. De plus, tous les 6 mois, les parents étaient contactés afin de préciser si leur enfant recevait des services scolaires et sociaux pour leurs difficultés de comportement. Le déroulement de cette recherche a été approuvé par le comité d'éthique en éducation et sciences sociales de l'Université de Sherbrooke (Québec, Canada).

\section{Instruments de mesure}

\section{Troubles du comportement perturbateur}

Le nombre de symptômes de TCP, incluant les symptômes du trouble oppositionnel (TOP) et ceux du trouble des conduites (TC), a été mesuré à l'aide de la version française de la Diagnostic Interview Schedule for 
Children-Revised (DISC-2.25) (Shaffer et al., 1993; version française de Breton, Bergeron, Valla, Berthiaume et St-Georges, 1998) auprès du parent et de l'enseignant. Un symptôme était présent s'il était rapporté par l'un ou l'autre des répondants. Le nombre total de symptômes de TCP (15 symptômes pour le trouble des conduites et 8 symptômes pour le trouble oppositionnel) a été utilisé comme variable à tous les temps de mesure. La version française du DISC-2.25 présente une bonne cohérence interne en ce qui concerne le trouble oppositionnel $(\alpha=0,80)$ et une cohérence acceptable pour le trouble des conduites $(\alpha=0,54)$ lorsque l'entrevue est réalisée auprès du parent (Breton et al., 1998). Ces informations ne sont pas disponibles lorsque l'entrevue est réalisée auprès de l'enseignant. Le DISC-2.25 permet, de prime abord, d'évaluer la présence de symptômes du TOP et du TC tels que décrits dans le DSM-III-R (APA, 1987), mais il a été adapté afin d'évaluer aussi les critères du DSM-IV-TR (APA, 2000). Les valeurs de Kappa calculées entre les versions du DSM-III-R et du DSM-IV-TR se sont avérées très bonnes, tant pour l'entrevue réalisée auprès du parent que celle réalisée auprès de l'enseignant (Déry et al., 2004).

\section{Caractéristiques individuelles}

Fonctions verbales et exécutives. Les habiletés sur le plan des fonctions verbales et exécutives ont été mesurées auprès de l'enfant par trois tests distincts. D'abord, le nombre de couleurs/mots lus correctement, tel que mesuré à partir du Stroop Color-Word Test (SCWT; Lezak, Howieson et Loring, 2004) a été utilisé comme indicateur d'impulsivité. Pour cette échelle, le coefficient de fidélité test-retest mesuré à une semaine d'intervalle est de 0,77 (Franzen, Tishelman, Sharp et Friedman, 1987). Ensuite, le score " copie + mémoire » de la figure complexe de Rey-Osterrieth (Rey, 1959) a été retenu comme variable à l'étude et informe à la fois sur les habiletés de planification et d'organisation ainsi que sur la mémoire à court terme. Les coefficients d'accord interjuges de cet instrument sont toujours supérieurs à 0,80 (Lezak et al., 2004). Dans la présente étude, les coefficients d'accord interjuges sont excellents, tant pour la copie que pour la mémoire $(\mathrm{k}=0,98)$. Enfin, l'Échelle de vocabulaire en image Peabody (ÉVIP; Dunn, Thériault-Whalen et Dunn, 1993) est spécifiquement utilisée pour évaluer les habiletés verbales. Cette échelle, évaluant l'étendue du vocabulaire réceptif des enfants, a été validée au Canada français et peut être utilisée à titre d'indicateur des habiletés cognitives des enfants $(\alpha=0,66$ à 0,88$)$. Le score standardisé selon l'âge et le genre obtenu avec 
l'ÉVIP corrèle fortement avec les échelles d'intelligence Stanford-Binet et Wechsler (Dunn et al., 1993).

Tempérament. Pour évaluer le tempérament des enfants, le parent répondant a complété la version française du Middle Childhood Temperament Questionnaire (MCTQ; Hegvik, McDevitt et Carey, 1982), qui permet d'évaluer neuf traits du tempérament : activité, régularité, approche/retrait, adaptabilité, intensité, humeur, persistance, distractibilité et réactivité. Les qualités psychométriques du MCTQ sont très bonnes, avec des coefficients de cohérence interne variant de 0,71 et 0,87 selon l'échelle et des coefficients tests-retest allant de 0,80 à 0,93 (Hegvik et al., 1982).

Intégration sociale. L'échelle de compétences sociales de la Child Behavior Checklist (Achenbach et Edelbrock, 1983) adaptée aux fins de l'EQSMJ (Valla et al., 1994) a été utilisée auprès du parent. Cette échelle documente le nombre d'organisations sociales et sportives dans lesquelles est impliqué l'enfant, le nombre d'amis proches et le nombre d'activités sociales auxquelles il participe, pour ensuite permettre le calcul d'un score reflétant la qualité de l'intégration sociale de l'enfant.

\section{Caractéristiques familiales}

Pratiques éducatives. Le manque de supervision parentale et l'inconstance disciplinaire des parents ont été évalués auprès du parent à l'aide de deux échelles de l'Alabama Parenting Questionnaire (Frick, 1991). Ces échelles, traduites en français pour les besoins de l'étude selon la méthode bidirectionnelle, comprennent dix et six items répondus sur une échelle de type Likert allant de 1 ("jamais») à 5 (" toujours»). Plus le score est élevé aux échelles du manque de supervision et de l'inconstance disciplinaire, plus ces pratiques sont lacunaires. Les deux échelles, lorsqu'utilisées auprès des parents, comportent des qualités psychométriques satisfaisantes. Leurs coefficients tests-retest sont respectivement de 0,66 et 0,85 et, pour la cohérence interne, ils sont de 0,67 pour les deux échelles (Shelton, Frick et Wooton, 1996).

Environnement criminogène. Pour déterminer si un membre de la famille (parents, fratrie) a eu des démêlés avec la justice en raison de comportements criminels, le parent a répondu à une question de type oui ou non : «Depuis la naissance de l'enfant, est-ce qu'un ou des membres de votre famille qui ont habité avec lui/elle a eu des démêlés avec la justice?». 
Santé mentale des parents. Pour évaluer la santé mentale des parents, la Composite International Diagnostic Interview Simplified (CIDIS; Fournier, Lesage, Toupin et Cyr, 1997), une version française abrégée et simplifiée de la Diagnostic Interview Schedule de Robins et al. (1985), a été utilisée auprès du parent. La CIDIS permet de diagnostiquer les troubles de santé mentale les plus fréquemment rencontrés dans la population à partir des critères du DSM-III-R (APA, 1987). Quatre prédicteurs reliés à la santé mentale des parents ont été retenus : la présence, au cours de la vie, d'un diagnostic d'anxiété généralisée, de dépression, d'abus ou de dépendance à l'alcool et d'abus ou de dépendance à la drogue. Fournier et al. (1997) indiquent une correspondance relativement satisfaisante entre les résultats à la CIDIS et la pose d'un diagnostic effectuée par un psychiatre $(k=0,70$ pour l'abus d'alcool; $k=0,42$ pour l'abus de drogues; $k=0,33$ pour la dépression et $k<0,33$ pour l'anxiété). Dans l'ensemble, il y a une bonne concordance entre la présence d'un diagnostic posé avec la CIDIS et la nécessité de recevoir des soins spécialisés (Fournier et al., 1997).

\section{Services reçus}

Pour documenter les services reçus, les parents ont répondu par téléphone, à tous les six mois au cours des deux premières années de suivi, à un questionnaire maison portant sur les services spécialisés reçus dans les six derniers mois. Le questionnaire, adapté de celui de l'EQSMJ (Valla et al., 1994), porte sur la présence et la fréquence (échelle ordinale en quatre points : "au moins une fois par semaine "; «au moins une fois par deux semaines »; « au moins une fois par mois »; «moins d'une fois par mois ») de services psychoéducatifs ou psychologiques octroyés à l'enfant ainsi que sur la présence de services obtenus d'un Centre jeunesse $e^{2}$ pour les problèmes de comportement de l'enfant. Ces données ont permis le calcul d'un score d'intensité des services psychoéducatifs ou psychologiques reçus au cours des deux premières années de suivi.

\section{Analyses}

Une analyse de classes de trajectoires latentes a été effectuée à l'aide du logiciel MPlus 5.1 (Muthén et Muthén, 1998-2008) afin d'identifier les trajectoires développementales des TCP dans les six années de suivis

\footnotetext{
$2 \quad$ Au Québec, les Centres jeunesse sont des organismes publics qui offrent des services sociaux spécialisés aux enfants, aux adolescents, aux mères et aux familles en difficulté (http://www.ciusss-capitalenationale.gouv.qc.ca/centre-jeunesse-de-quebec-institutuniversitaire).
} 
(aux 4 temps de mesure). L'avantage de cette analyse est qu'elle permet de gérer les données manquantes. Plusieurs modèles ont été vérifiés, en commençant par un modèle à une classe jusqu'au modèle à 5 classes. Le modèle retenu est celui qui a obtenu la plus petite valeur du Bayesan Information Criterion (BIC; Raftery, 1995) et une valeur d'entropie qui se rapproche davantage de 1 (McLachlan et Peel, 2000). Linvariance du modèle de trajectoire selon le genre a été testée : les indices pour juger de la qualité des modèles unisexe (garçons et filles simultanément) et multi-groupes (garçons et filles séparées) ont été estimés et leur BIC a été comparé. Un modèle à quatre trajectoires a été retenu (voir la section résultats). Toutefois, pour répondre à l'objectif de l'étude, seules les trajectoires des troubles persistants et décroissants ont été prises en compte.

Afin de déterminer les caractéristiques individuelles et familiales qui permettent de prédire l'appartenance à la trajectoire persistante par rapport à la trajectoire décroissante et de vérifier l'effet modérateur du genre, les analyses ont été réalisées en deux étapes. Ces analyses ont été effectuées avec le logiciel SPSS 17 (SPSS Inc., 2008). D'abord, une série de régressions logistiques a été réalisée pour vérifier la contribution unique de chaque caractéristique et l'effet modérateur du genre sur chacune de ces associations. Lintensité des services reçus ainsi que l'âge des enfants ont été insérés comme covariables dans ces analyses. Ensuite, les caractéristiques pour lesquelles le seuil de signification s'est avéré inférieur ou égal à $p \leq 0,2$ soit en effet principal ou en modération avec le genre ont été retenues comme prédicteurs à inclure dans la régression logistique multivariée ( $2^{\mathrm{e}}$ étape). Dans cette analyse multivariée, le score d'intensité des services reçus ainsi que l'âge des enfants ont été insérés comme variables de contrôle dans un premier bloc. Les prédicteurs retenus à l'étape 1 ont été insérés dans le deuxième bloc. Le genre ainsi que les interactions entre chacun des prédicteurs retenus ont finalement été insérés dans le troisième bloc. Préalablement à cette régression, les corrélations ont été calculées entre chacune des variables pour s'assurer de l'absence de multicolinéarité. Les prédicteurs ont été standardisés (centrés à la moyenne) afin de réduire les corrélations entre ces prédicteurs, le modérateur et leurs interactions (Cohen, Cohen, West et Aiken, 2003). 


\section{Résultats}

\section{Identification des trajectoires}

Le modèle à quatre trajectoires a été retenu puisqu'il présentait la plus petite valeur de Bayesan Information Criterion (BIC; Raftery, 1995) et une valeur d'entropie acceptable se rapprochant davantage de 1 (McLachlan et Peel, 2000). Les valeurs du BIC pour les modèles de deux à cinq trajectoires sont respectivement de 4484, 4475, 4447 et 4454, et les valeurs d'entropie sont de $0,662,0,646,0,708$ et 0,716 . Les résultats, quant à l'invariance de ce modèle à quatre trajectoires selon le genre, montrent qu'il n'est pas justifié de traiter séparément les garçons et les filles, puisque la valeur du BIC obtenue pour le modèle unigenre (garçons et filles simultanément) est plus petite $(\mathrm{BIC}=4447)$ que celle obtenue pour le modèle multi-groupes (garçons et filles séparément : $\mathrm{BIC}=4815$ ). Comme il n'y a pas amélioration de la qualité du modèle en traitant les garçons et les filles séparément, on peut conclure que ces différentes trajectoires s'appliqueraient tant aux filles qui présentent des TCP précoces qu'aux garçons. Chacune des trajectoires est illustrée à la figure 1 .

Figure 1

Trajectoires des troubles de comportement perturbateur chez les garçons et chez les filles.
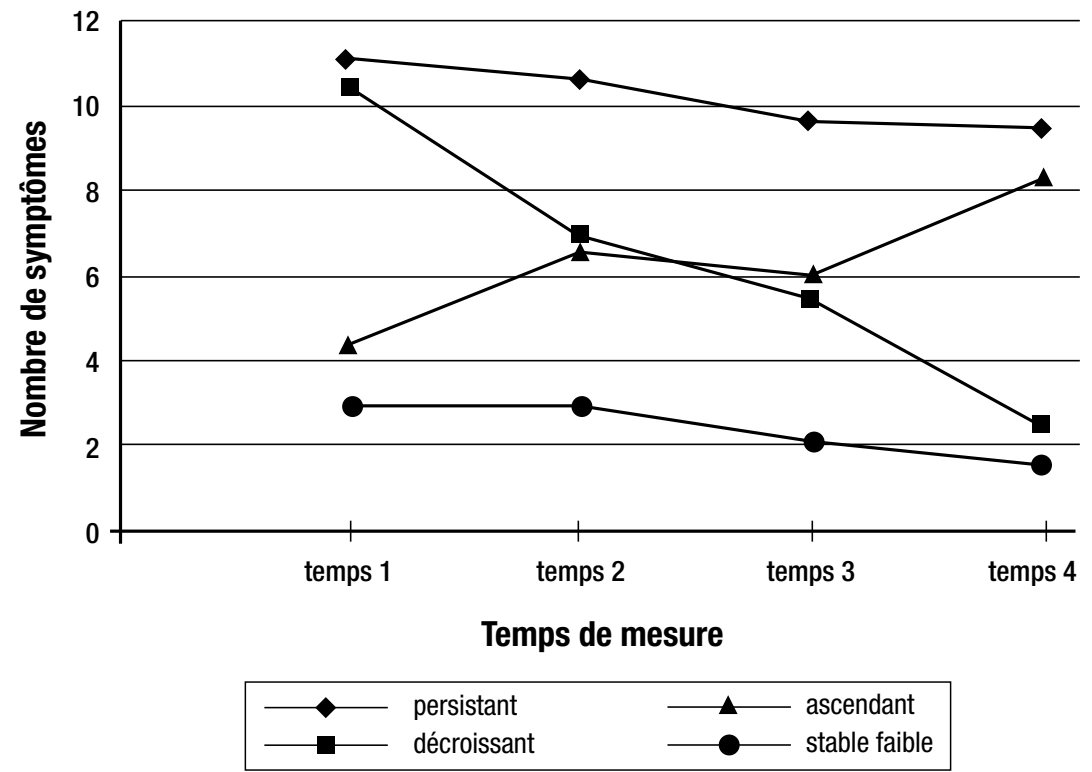
Ainsi, la première trajectoire regroupe les enfants qui présentent des TCP persistants ( $n=33 ; 24 \%$ de filles) et correspond à $12,3 \%$ de l'échantillon. Le nombre moyen de symptômes de TCP présentés au temps 1 par les enfants de la trajectoire persistante est de 11,2 (é.-t. = 2,3). Six ans plus tard, ces jeunes présentent encore 9,5 (é.-t. $=2,5)$ symptômes en moyenne. La seconde trajectoire inclut les enfants dont les troubles sont décroissants ( $n=87 ; 30 \%$ de filles) et représente $32,5 \%$ de l'échantillon. Ces enfants présentent, en moyenne, 10,4 (é.-t. $=2,5$ ) symptômes de TCP au temps 1 , mais seulement 2,5 $(e ́$. $-t=2,0)$ symptômes six ans plus tard (temps 4$)$. Il n'y a pas de différences significatives entre les enfants de ces deux trajectoires au niveau de l'âge, $t(118)=1,173 ; p=0,243$, du genre, $\chi^{2}(1)=0,375$; $p=0,540$, et du nombre de symptômes de TCP présentés au premier temps de mesure, $t(116)=-1,493 ; p=0,138$. Quant à l'intensité des services spécialisés reçus par les enfants appartenant à ces deux trajectoires, il s'avère que les enfants de la trajectoire persistante ont reçu, en moyenne, plus de services au cours des deux premières années de suivi que ceux de la trajectoire décroissante, $t(117)=-3,207$; $p=0,000$. Toutefois, il n'y a aucune différence significative entre ces deux trajectoires sur l'intensité moyenne de services reçus dans les années subséquentes (de 2 à 4 ans : $t(101)=-0,941 ; p=0,349$; de 4 à 6 ans : $t(101)=-0,849 ; p=0,398)$. Ces résultats portent à croire que les services sont possiblement ajustés selon l'ampleur des TCP présentés au départ, mais non en fonction de leur persistance. Donc, uniquement l'intensité des services reçus au cours des deux premières années de suivi sera utilisée comme covariable dans les analyses.

\section{Caractéristiques individuelles et familiales associées aux trajectoires}

Les données descriptives pour l'ensemble des variables à l'étude sont présentées selon le genre et la trajectoire d'appartenance (persistante, décroissante) dans le tableau 1. Dans l'ensemble et tel que mentionné précédemment, peu importe que les enfants appartiennent à la trajectoire persistante ou décroissante, ils sont nombreux à vivre dans un environnement criminalisé $(8 \%$ à $21 \%)$ où les problèmes de santé mentale chez les parents sont fréquents ( $8 \%$ à $52 \%$ ) et où la discipline parentale manque de constance (moyenne allant de 2,6 à 3,$1 ; M=2,7$; é.-t. $=0,8)$. 


\section{Tableau 1}

\section{Données descriptives des variables mesurées au temps 1 selon la trajectoire et le genre}

\begin{tabular}{|c|c|c|c|c|c|c|c|}
\hline \multirow{3}{*}{ Caractéristiques } & \multicolumn{3}{|c|}{ Trajectoire décroissante } & \multicolumn{3}{|c|}{ Trajectoire persistante } & \\
\hline & $\begin{array}{l}\text { Garçons } \\
(n=61)\end{array}$ & $\begin{array}{l}\text { Filles } \\
(n=26)\end{array}$ & $\begin{array}{l}\text { Total } \\
(n=87)\end{array}$ & $\begin{array}{c}\text { Garçons } \\
(n=25)\end{array}$ & $\begin{array}{l}\text { Filles } \\
(n=8)\end{array}$ & $\begin{array}{l}\text { Total } \\
(n=33)\end{array}$ & \\
\hline & $M(e ́ .-t)$. & $M$ (é.-t.) & $M$ (é.-t.) & $M$ (é.-t.) & $M(e ́$-t.t) & $M(e ́ .-t)$. & Étendue \\
\hline \multicolumn{8}{|c|}{ Caractéristiques individuelles } \\
\hline \multicolumn{8}{|c|}{ Fonctions verbales et exécutives } \\
\hline Couleurs/mots (SCWT) & $\begin{array}{l}23,9 \\
(8,1)\end{array}$ & $\begin{array}{c}22,1 \\
(11,7)\end{array}$ & $\begin{array}{l}23,4 \\
(9,3)\end{array}$ & $\begin{array}{l}21,3 \\
(8,5)\end{array}$ & $\begin{array}{l}18,6 \\
(7,7)\end{array}$ & $\begin{array}{l}20,7 \\
(8,3)\end{array}$ & 0 à 63 \\
\hline $\begin{array}{l}\text { Copie+mémoire } \\
\text { (figure de Rey) }\end{array}$ & $\begin{array}{c}37,8 \\
(16,3) \\
\end{array}$ & $\begin{array}{c}31,7 \\
(15,6)\end{array}$ & $\begin{array}{c}36,0 \\
(16,2)\end{array}$ & $\begin{array}{c}30,1 \\
(15,5)\end{array}$ & $\begin{array}{c}32,1 \\
(14,9)\end{array}$ & $\begin{array}{c}30,6 \\
(15,1) \\
\end{array}$ & 0,5 à 63 \\
\hline Habiletés verbales (ÉVIP) & $\begin{array}{l}108,7 \\
(12,1) \\
\end{array}$ & $\begin{array}{l}107,2 \\
(16,8) \\
\end{array}$ & $\begin{array}{l}108,3 \\
(13,6) \\
\end{array}$ & $\begin{array}{l}112,5 \\
(11,0) \\
\end{array}$ & $\begin{array}{c}97,3 \\
(16,8) \\
\end{array}$ & $\begin{array}{l}109,2 \\
(13,7) \\
\end{array}$ & 69 à 141 \\
\hline \multicolumn{8}{|l|}{ Tempérament } \\
\hline Activité (MCTQ) & $3,8(0,9)$ & $3,8(0,9)$ & $3,8(0,9)$ & $4,0(0,9)$ & $4,1(1,4)$ & $4,0(1,0)$ & 1,8 à 5,7 \\
\hline Régularité (MCTQ) & $3,4(0,6)$ & $3,5(0,8)$ & $3,5(0,7)$ & $3,4(0,7)$ & $3,8(0,8)$ & $3,5(0,8)$ & 1,9 à 5,0 \\
\hline Approche-retrait (MCTQ) & $2,7(0,7)$ & $2,9(1,0)$ & $2,7(0,8)$ & $2,7(0,8)$ & $2,7(0,6)$ & $2,7(0,7)$ & 1,0 à 5,5 \\
\hline Adaptabilité (MCTQ) & $3,8(0,6)$ & $3,9(0,5)$ & $3,8(0,6)$ & $3,9(0,8)$ & $3,8(0,5)$ & $3,8(0,8)$ & 2,2 à 5,3 \\
\hline Intensité (MCTQ) & $4,0(0,6)$ & $4,2(0,7)$ & $4,0(0,6)$ & $4,2(0,8)$ & $4,2(0,8)$ & $4,2(0,8)$ & 2,6 à 5,7 \\
\hline Humeur (MCTQ) & $3,7(0,7)$ & $4,0(0,5)$ & $3,8(0,6)$ & $3,8(0,8)$ & $4,1(0,7)$ & $3,9(0,8)$ & 2,0 à 5,5 \\
\hline Persistance (MCTQ) & $3,7(0,7)$ & $3,6(0,8)$ & $3,7(0,7)$ & $3,5(0,7)$ & $3,6(1,1)$ & $3,5(0,8)$ & 1,9 à 5,4 \\
\hline Distractibilité (MCTQ) & $4,3(0,8)$ & $4,6(0,8)$ & $4,4(0,8)$ & $4,5(0,9)$ & $4,8(0,8)$ & $4,6(0,9)$ & 2,6 à 6,0 \\
\hline Réactivité (MCTQ) & $3,9(0,6)$ & $3,8(0,6)$ & $3,9(0,6)$ & $3,9(0,7)$ & $4,3(1,1)$ & $4,0(0,8)$ & 2,3 à 5,9 \\
\hline Intégration sociale & $5,5(3,8)$ & $5,2(3,9)$ & $5,4(3,8)$ & $6,4(3,1)$ & $4,6(2,2)$ & $6,0(3,0)$ & 0 à 15 \\
\hline \multicolumn{8}{|c|}{ Caractéristiques familiales } \\
\hline \multicolumn{8}{|l|}{ Pratiques éducatives } \\
\hline Manque de supervision & $1,6(0,5)$ & $1,5(0,4)$ & $1,5(0,4)$ & $1,5(0,6)$ & $1,7(0,9)$ & $1,6(0,7)$ & 1,0 à 3,2 \\
\hline \multirow[t]{2}{*}{ Discipline inconstante } & $2,8(0,6)$ & $3,1(0,5)$ & $2,9(0,6)$ & $2,6(0,7)$ & $3,0(0,9)$ & $2,7(0,8)$ & 1,5 à 4,3 \\
\hline & $\%$ & $\%$ & $\%$ & $\%$ & $\%$ & $\%$ & Étendue \\
\hline Criminalité & 3 & 19 & 8 & 20 & 25 & 21 & 0 à 1 \\
\hline \multicolumn{8}{|l|}{ Santé mentale des parents } \\
\hline Dx anxiété & 8 & 8 & 8 & 20 & 13 & 18 & 0 à 1 \\
\hline Dx dépression & 52 & 38 & 48 & 40 & 38 & 39 & 0 à 1 \\
\hline Dx alcool & 13 & 12 & 13 & 16 & 0 & 12 & 0 à 1 \\
\hline Dx drogue & 13 & 23 & 16 & 32 & 25 & 30 & 0 à 1 \\
\hline
\end{tabular}

Note $: \mathrm{Dx}=$ diagnostic 


\section{Tableau 2}

\section{Régressions logistiques réalisées par caractéristiques prédisant l'appartenance à la trajectoire persistante : effet modérateur du genre}

\begin{tabular}{|c|c|c|c|c|c|c|}
\hline & \multicolumn{2}{|c|}{ Caractéristiques $^{\mathrm{a}}$} & \multicolumn{2}{|c|}{ Genre $^{b}$} & \multicolumn{2}{|c|}{$\begin{array}{c}\text { Caractéristiques } \\
\text { genre }\end{array}$} \\
\hline & $\beta$ & $p$ & $\beta$ & $p$ & $\beta$ & $p$ \\
\hline \multicolumn{7}{|c|}{ Caractéristiques individuelles } \\
\hline \multicolumn{7}{|c|}{ Fonctions verbales et exécutives } \\
\hline Couleurs/mots (SCWT) & $-0,043$ & 0,186 & $-0,153$ & 0,905 & $-0,015$ & 0,791 \\
\hline $\begin{array}{l}\text { Copie+mémoire } \\
\text { (figure de Rey) }\end{array}$ & $-0,033$ & 0,079 & $-1,299$ & 0,293 & 0,030 & 0,388 \\
\hline Habiletés verbales (ÉVIP) & 0,028 & 0,218 & 7,572 & 0,050 & $-0,076$ & 0,040 \\
\hline \multicolumn{7}{|l|}{ Tempérament } \\
\hline Activité (MCTQ) & 0,307 & 0,323 & $-0,285$ & 0,898 & 0,003 & 0,996 \\
\hline Régularité (MCTQ) & 0,059 & 0,886 & $-1,426$ & 0,588 & 0,305 & 0,664 \\
\hline Approche-retrait (MCTQ) & 0,263 & 0,491 & 1,182 & 0,536 & $-0,520$ & 0,431 \\
\hline Adaptabilité (MCTQ) & 0,402 & 0,303 & 1,378 & 0,697 & $-0,429$ & 0,691 \\
\hline Intensité (MCTQ) & 0,559 & 0,168 & 1,614 & 0,603 & $-0,463$ & 0,527 \\
\hline Humeur (MCTQ) & 0,322 & 0,413 & $-2,063$ & 0,552 & 0,431 & 0,617 \\
\hline Persistance (MCTQ) & $-0,423$ & 0,268 & $-2,076$ & 0,393 & 0,505 & 0,444 \\
\hline Distractibilité (MCTQ) & 0,377 & 0,253 & $-2,348$ & 0,454 & 0,426 & 0,518 \\
\hline Réactivité (MCTQ) & $-0,007$ & 0,988 & $-4,491$ & 0,155 & 1,051 & 0,170 \\
\hline Intégration sociale & 0,088 & 0,216 & 0,526 & 0,560 & $-0,148$ & 0,318 \\
\hline \multicolumn{7}{|c|}{ Caractéristiques familiales } \\
\hline \multicolumn{7}{|l|}{ Pratiques éducatives } \\
\hline Manque de supervision & 0,077 & 0,882 & $-1,854$ & 0,219 & 1,029 & 0,260 \\
\hline Discipline inconstante & $-0,303$ & 0,461 & $-1,157$ & 0,665 & 0,321 & 0,718 \\
\hline Criminalité & 1,828 & 0,041 & $-0,332$ & 0,553 & $-0,524$ & 0,705 \\
\hline \multicolumn{7}{|l|}{ Santé mentale des parents } \\
\hline Dx anxiété & 1,137 & 0,117 & $-0,176$ & 0,740 & $-0,732$ & 0,637 \\
\hline Dx dépression & $-0,824$ & 0,123 & $-0,525$ & 0,418 & 0,430 & 0,685 \\
\hline Dx alcool & 0,219 & 0,759 & $-0,120$ & 0,815 & $-20,368$ & 0,999 \\
\hline Dx drogue & 1,045 & 0,086 & $-0,238$ & 0,678 & $-0,215$ & 0,855 \\
\hline
\end{tabular}

Note : Lintensité des services reçus et l'âge des enfants ont été contrôlés dans les analyses. ${ }^{a}$ prédit la probabilité d'appartenir à la trajectoire persistante (par rapport à la décroissante). ${ }^{\mathrm{b}}$ filles $=1$ et garçons $=0$, on prédit donc les filles par rapport aux garçons. Dx = diagnostic 
Les analyses de régression réalisées séparément pour chacune des caractéristiques individuelles et familiales (étape 1) ont permis d'identifier un total de neuf caractéristiques qui présentent un plus grand potentiel $(p \leq 0,2)$ pour prédire l'appartenance à la trajectoire persistante. Les résultats obtenus sont présentés dans le tableau 2. Sur le plan des caractéristiques individuelles, on retrouve les habiletés verbales, l'impulsivité (nombre de couleurs/mots lus), les habiletés d'organisation et de planification incluant la mémoire à court terme (score copie+mémoire) ainsi que deux échelles du tempérament (intensité, réactivité). Une seule de ces variables individuelles s'avère significative $(p \leq 0,05)$. En effet, des habiletés verbales plus faibles sont particulièrement associées à la trajectoire persistante des filles. Quant aux caractéristiques familiales, quatre variables ont été retenues $(p \leq 0,2)$. Ce sont la présence de trois troubles de santé mentale chez le parent, soit l'anxiété généralisée, la dépression majeure et l'abus ou la dépendance à la drogue, ainsi que la présence de criminalité dans la famille. La criminalité des parents serait significativement associée à l'appartenance à la trajectoire persistante, et ce, pour l'ensemble des participants sans égard au genre.

\section{Tableau 3}

\section{Corrélations entre les caractéristiques individuelles et familiales retenues à l'étape 1}

\begin{tabular}{|c|c|c|c|c|c|c|c|c|c|c|c|c|}
\hline Caractéristiques & 1 & 2 & 3 & 4 & 5 & 6 & 7 & 8 & 9 & 10 & 11 & 12 \\
\hline 1. Couleurs/mots & - & & & & & & & & & & & \\
\hline 2. Copie+mémoire & $0,24^{\star \star}$ & - & & & & & & & & & & \\
\hline $\begin{array}{l}\text { 3. Habiletés } \\
\text { verbales }\end{array}$ & 0,08 & 0,01 & - & & & & & & & & & \\
\hline 4. Intensité & $-0,22^{*}$ & $-0,05$ & $-0,02$ & - & & & & & & & & \\
\hline 5. Réactivité & $-0,14$ & $-0,05$ & $-0,11$ & $0,49^{\star \star \star}$ & 一 & & & & & & & \\
\hline 6. Criminalité & $-0,29^{\star \star}$ & $-0,10$ & $-0,09$ & 0,18 & 0,04 & - & & & & & & \\
\hline $\begin{array}{l}\text { 7. Dx anxiété } \\
\text { parents }\end{array}$ & $-0,14$ & $-0,02$ & $-0,12$ & 0,09 & 0,18 & 0,12 & - & & & & & \\
\hline $\begin{array}{l}\text { 8. Dx dépression } \\
\text { parents }\end{array}$ & 0,03 & 0,06 & 0,13 & 0,14 & $0,21^{*}$ & $-0,02$ & $0,38^{\star \star \star}$ & - & & & & \\
\hline $\begin{array}{l}\text { 9. Dx drogue } \\
\text { parents }\end{array}$ & $-0,17$ & 0,03 & 0,03 & $-0,04$ & 0,01 & $0,40^{\star \star \star}$ & 0,16 & 0,17 & - & & & \\
\hline 10. Genre & $-0,09$ & $-0,11$ & $-0,16$ & 0,11 & $-0,00$ & 0,18 & $-0,04$ & $-0,10$ & 0,06 & - & & \\
\hline \begin{tabular}{|l}
$\begin{array}{l}\text { 11. Fréquence } \\
\text { des services }\end{array}$ \\
\end{tabular} & 0,06 & $-0,05$ & 0,08 & -0.02 & 0,09 & $-0,09$ & 0,02 & 0,15 & $-0,02$ & $-0,04$ & - & \\
\hline 12. Âge & $-0,28^{\star \star}$ & $0,61^{* \star \star}$ & $-0,32^{\star \star \star}$ & $-0,07$ & 0,12 & $-0,01$ & $-0,01$ & $-0,03$ & 0,07 & $-0,07$ & $-0,18^{\star}$ & - \\
\hline
\end{tabular}

Note $: \mathrm{Dx}=$ diagnostic. ${ }^{*} p<, 05 ; \quad * * p<, 01 ; * * * p<, 001$ 


\section{Tableau 4}

\section{Régression logistique multivariée prédisant l'appartenance à la trajectoire persistante ${ }^{\mathrm{a}}$ : effet modérateur du genre}

\begin{tabular}{|c|c|c|c|c|c|}
\hline Caractéristiques & $\beta$ & $S E$ & $p$ & $O R^{a}$ & $95 \%$ IC \\
\hline$\chi^{2}=11,42$ & \multicolumn{5}{|c|}{$p=0,003 \quad R^{2}=.143$} \\
\hline Intensité des services & 0,76 & 0,26 & 0,003 & 2,14 & 1,29 à 3,55 \\
\hline Âge & $-0,08$ & 0,13 & 0,550 & 0,93 & 0,73 à 1,19 \\
\hline$\chi^{2}=27,0$ & \multicolumn{5}{|c|}{$p=0,005 \quad R^{2}=.315$} \\
\hline \multicolumn{2}{|l|}{ Couleurs/mots (SCWT) } & 0,27 & 0,458 & 0,82 & 0,48 à 1,39 \\
\hline Copie+mémoire (figure de Rey) & $-0,29$ & 0.33 & 0,386 & 0,75 & 0,39 à 1,44 \\
\hline Habiletés verbales (ÉVIP) & 0,29 & 0,26 & 0,272 & 1,34 & 0,80 à 2,24 \\
\hline Intensité (MCTQ) & 0.20 & 0,34 & 0,555 & 1,22 & 0,63 à 2,39 \\
\hline Réactivité (MCTQ) & $-0,03$ & 0,33 & 0,930 & 0,97 & 0,51 à 1,84 \\
\hline Criminalité & 0,93 & 0,80 & 0,244 & 2,54 & 0,53 à 12,20 \\
\hline Dx anxiété parents & 1,78 & 0,88 & 0,044 & 5,91 & 1,05 à 33,40 \\
\hline Dx dépression parents & $-1,41$ & 0,63 & 0,024 & 0,24 & 0,07 à 0,83 \\
\hline Dx drogue parents & 0.48 & 0,70 & 0,491 & 1,61 & 0,41 à 6,31 \\
\hline \multicolumn{6}{|l|}{ Bloc $3 \quad \chi^{2}=43,47$} \\
\hline Genre $^{b}$ & $-1,02$ & 1,10 & 0,355 & 0,36 & 0,04 à 3,11 \\
\hline Couleurs/mots $($ SCWT $) \times$ genre & $-2,01$ & 1,11 & 0,070 & 0,13 & 0,02 à 1,18 \\
\hline $\begin{array}{l}\text { Copie+mémoire } \\
\text { (figure de Rey) } \times \text { genre }\end{array}$ & 0,94 & 1,05 & 0,369 & 2,56 & 0,33 à 19,86 \\
\hline Habiletés verbales $($ ÉVIP) $\times$ genre & $-1,02$ & 0,69 & 0,144 & 0,36 & 0,09 à 1,41 \\
\hline Intensité $(\mathrm{MCTQ}) \times$ genre & $-1,83$ & 0,98 & 0,063 & 0,16 & 0,02 à 1,11 \\
\hline Réactivité $(\mathrm{MCTQ}) \times$ genre & 2,08 & 1,04 & 0,046 & 7,96 & 1,04 à 61,0 \\
\hline Criminalité $\times$ genre & $-0,70$ & 2,28 & 0,759 & 0,50 & 0,01 à 42,97 \\
\hline Dx anxiété parents $\times$ genre & $-4,86$ & 3,06 & 0,112 & 0,01 & 0,00 à 3,10 \\
\hline Dx dépression parents $\times$ genre & 0,99 & 2,08 & 0,635 & 2,69 & 0,05 à 159,25 \\
\hline Dx drogue parents $\times$ genre & 0,96 & 1,76 & 0,586 & 2,61 & 0,08 à 82,25 \\
\hline
\end{tabular}

Note $:$ IC $=$ intervalle de confiance pour les odds ratio $(\mathrm{OR})$. Lintensité des services reçus a été contrôlée dans les analyses. ${ }^{a}$ prédit la probabilité d'appartenir à la trajectoire persistante (par rapport à la décroissante). ${ }^{\mathrm{b}}$ filles $=1$ et garçons $=0$, on prédit donc les filles par rapport aux garçons. Dx = diagnostic

Le tableau 3 montre que les corrélations calculées entre chacune des neuf caractéristiques retenues à l'étape 1 sont toutes inférieures à 
0,5. On peut convenir qu'elles ne sont pas suffisamment élevées pour représenter un risque de multicolinéarité. Ces neuf variables ainsi que le genre et leur interaction ont donc été inclus dans le modèle de régression logistique multivariée subséquent (étape 2 ).

Les résultats de cette dernière analyse sont présentés dans le tableau 4. Au-delà de l'intensité des services reçus et de l'âge des enfants $\left(R^{2}=0,143\right)$, l'ajout des prédicteurs dans le deuxième bloc permet d'expliquer $31,5 \%$ de variance $\left(R^{2}=0,315\right)$. Deux prédicteurs s'avèrent significatifs dans le modèle : la présence d'un trouble d'anxiété, ainsi que l'absence de dépression chez les parents, sont associées à l'appartenance à la trajectoire persistante. En ajoutant le genre et ses interactions avec les prédicteurs dans un troisième bloc, on explique $47,3 \%$ de variance $\left(R^{2}=0,473\right)$. Au-delà de l'effet d'anxiété et de dépression des parents qui demeurent significatifs, il y a ajout d'une interaction significative du tempérament réactif avec le genre. Ainsi, plus les filles ont un tempérament réactif, plus elles risquent d'appartenir à la trajectoire persistante. Cet effet du tempérament réactif ne s'observe pas chez les garçons. Bien qu'il y ait quelques effets marginalement significatifs $(p<0,1)$, aucun des autres prédicteurs inclus dans le modèle multivarié ne contribue de manière claire à distinguer le risque d'appartenir à la trajectoire persistante par rapport à la trajectoire décroissante.

\section{Discussion}

Cette étude visait à identifier les caractéristiques individuelles et familiales associées aux enfants, garçons et filles, présentant des TCP persistants par rapport à ceux dont les troubles ont décru. Cela, afin de planifier des stratégies d'intervention plus ciblées visant à limiter les conséquences éventuelles associées à la persistance de ces troubles. Comparativement à d'autres études ayant abordé la question, celle-ci a l'avantage de tenir compte de l'équivalence du nombre de symptômes de TCP présentés initialement entre les trajectoires comparées (persistante et décroissante) et de contrôler l'intensité des services reçus par les enfants, en plus de vérifier l'effet modérateur du genre.

On sait que les troubles survenant en bas âge sont plus à risque d'être persistants et envahissants (APA, 2013; Biederman et al., 2008; Lahey et al., 2005; Renk, 2008; Whittinger, et al., 2007). Or, des études antérieures ont indiqué que ces troubles diminuent considérablement chez plusieurs enfants (Barker et Maughan, 2009; Hoeve et al., 2008; Moffitt et al., 1996; Pitzer et al., 2010; Raine et al., 2005; Van Domburgh 
et al., 2009; Veenstra et al., 2009). Conformément à diverses études portant sur les trajectoires des TCP, et ce, peu importe que l'étude ait été réalisée auprès d'un échantillon clinique (Pitzer et al., 2010; Raine et al., 2005) ou populationnel (Barker et Maughan, 2009), quatre trajectoires développementales ont aussi été identifiées dans la présente étude : la trajectoire des TCP persistants, celle dont les troubles ont décru, celle où les troubles se sont accrus et celle dont les troubles sont demeurés faibles à tous les temps de mesure. À l'instar d'autres études (Barker et Maughan, 2009; Hoeve et al., 2008; Moffitt et al., 1996; Pitzer et al., 2010; Raine et al., 2005; Van Domburgh et al., 2009; Veenstra et al., 2009), un nombre considérable d'enfants appartient à la trajectoire décroissante $(32,5 \%)$. Et, parmi les 120 enfants qui présentaient un nombre de symptômes de TCP suffisamment élevé au temps 1 pour appartenir aux trajectoires décroissante ou persistante (plus de 10 symptômes en moyenne), $73 \%$ verront une décroissance considérable de leurs troubles. En outre, tout comme l'étude de Barker et Maughan (2009), les résultats portant sur l'invariance du modèle à quatre trajectoires selon le genre révèlent que les quatre trajectoires s'appliqueraient tant aux garçons qu'aux filles. Le nombre de filles est moins élevé que le nombre de garçons, mais la proportion des genres distribuée dans chacune des trajectoires est similaire. Ainsi, ces résultats indiquent que, en moyenne, le nombre de symptômes de TCP présentés par les filles est similaire à celui des garçons au départ et qu'il évoluera de manière comparable à celui des garçons au cours des six années de l'étude. Jusqu'ici, en s'appuyant sur ces résultats, on peut penser que les interventions pour prévenir la persistance des TCP devraient être les mêmes pour les garçons et pour les filles.

Toutefois, les résultats concernant l'identification des caractéristiques spécifiquement associées à l'une ou l'autre des trajectoires entraînent des recommandations un peu différentes. De nombreuses caractéristiques, touchant à la fois les vulnérabilités propres à l'enfant, la qualité de son intégration sociale, les vulnérabilités des parents et celles de l'environnement, ont été étudiées et permettent de dresser un portrait relativement complet des caractéristiques potentiellement discriminantes (Barker et Maughan, 2009; Hoeve et al., 2008; Lahey et al., 1999; Lahey et Waldman, 2003; Moffit, 1993; Moffitt, 2006; Moffitt et al., 1996 ; Pitzer et al., 2010; Raine et al., 2005; Van Domburgh et al., 2009; Veenstra et al., 2009). À la première étape des analyses de régression, deux caractéristiques contribuent de manière significative. Les filles de la trajectoire persistante ont moins d'habiletés verbales 
que les filles de la trajectoire décroissante, ce qui ne s'observe pas chez les garçons. Et, les enfants de la trajectoire persistante seraient plus nombreux à vivre dans un milieu criminogène. Les résultats de Veenstra et al. (2009) vont dans ce sens et indiquent que les parents des enfants dont les troubles persistent seraient davantage antisociaux. Ce résultat pourrait suggérer, d'une part, qu'il y a transmission intergénérationnelle possible des conduites de cette nature (Huesmann, Eron, Lefkowitz et Walder, 1984) et, d'autre part, que les parents présentant eux-mêmes des difficultés de cet ordre seraient plus limités pour faire face aux comportements problématiques de leurs enfants (Snyder et Patterson, 1987).

En plus d'avoir pris en compte un grand nombre de caractéristiques, la présente étude a l'avantage d'avoir recours à une analyse multivariée pour évaluer leur contribution additive et identifier celles qui discriminent plus fortement l'appartenance à l'une ou l'autre des trajectoires. À cet effet, lorsque les caractéristiques les plus importantes (couleurs/mots, copie+mémoire, habiletés verbales, intensité, réactivité, criminalité, diagnostics d'anxiété, de dépression et d'abus/dépendance à la drogue des parents) sont incluses dans une même analyse multivariée (étape 2), les caractéristiques qui ressortent significatives diffèrent. Avec l'analyse multivariée, on voit, entre autres, apparaître un effet combiné des problèmes de santé mentale des parents (plus fréquemment anxieux, mais moins fréquemment dépressifs) alors que la criminalité des parents ne ressort plus significativement une fois les autres caractéristiques individuelles et familiales prises en compte. Utilisant un devis de recherche comparable sur le plan de l'âge des enfants et des moments de collecte de données, des résultats similaires sont obtenus dans l'étude de Barker et Maughan (2009). Si, dans leurs analyses univariées, la présence de démêlés avec la justice ressortait significativement associée à la trajectoire persistante, leur analyse multivariée réalisée auprès de l'ensemble des enfants (garçons et filles) dévoile plutôt l'importance de la présence d'anxiété chez la mère. Éventuellement on pourrait vérifier si la santé mentale de la mère, et peut-être davantage l'anxiété, serait un médiateur des liens entre la présence de criminalité dans la famille et la persistance des TCP des enfants. Dans un tel cas, des services en santé mentale offerts spécifiquement aux parents seraient possiblement à prioriser.

Lors des analyses univariées (étape 1), les filles de la trajectoire persistante ressortaient comme étant particulièrement vulnérables sur le plan des habiletés verbales. L’analyse multivariée (étape 2) révèle 
plutôt que les filles de la trajectoire persistante sont plus susceptibles d'avoir un tempérament réactif. Avec la contribution des autres caractéristiques individuelles et familiales, l'analyse multivariée permet de mettre à l'avant-plan l'importance de ce trait de caractère pour les filles. Les modèles explicatifs (Lahey, Waldman et McBurnett, 1999; Lahey et Waldman, 2003; Moffitt, 1993, 2006; Moffitt et al., 1996) et les études empiriques relevaient aussi le caractère plus vulnérable des enfants de la trajectoire persistante. D'ailleurs, les résultats de Baker et Maughan (2009), ainsi que ceux de Veenstra et al. (2009), indiquent que les enfants dont les troubles persistent ont un tempérament plus difficile. Notre étude révèle que cette vulnérabilité tempéramentale associée à la trajectoire persistante, tel qu'avoir un caractère réactif, concernerait surtout les filles. Comme ce trait de caractère semble plus commun chez les garçons et s'observe plus rarement chez les filles (Moffitt, 1993; Moffitt et al., 1996), cette variable a probablement un potentiel de discrimination plus grand (une variance plus élevée) pour distinguer des sous-groupes de filles.

D'autres études appuient aussi l'idée que les filles appartenant à la trajectoire persistante ont plusieurs vulnérabilités : elles présenteraient davantage de symptômes du TDAH que les filles dont les troubles ont décru (Pitzer et al., 2010), elles seraient davantage confrontées à l'utilisation de pratiques éducatives sévères (Barker et Maughan, 2009) et elles seraient plus souvent témoins de violence conjugale (Barker et Maughan, 2009). Bien que les connaissances soient encore limitées quant à l'identification des vulnérabilités précises qui caractérisent les filles, les résultats obtenus appuient la pertinence de poursuivre l'étude des facteurs associés à la persistance des troubles du comportement propre aux filles et aux garçons.

Enfin, dans l'ensemble et malgré un nombre considérable de caractéristiques étudiées, nos résultats montrent plutôt que peu de vulnérabilités, tant individuelles que familiales, contribuent significativement à prédire l'appartenance à la trajectoire persistante. Rappelons que l'échantillon regroupe des jeunes âgés de 6 à 13 ans, ayant donc jusqu'à 6 ans d'écart au premier temps de mesure. Bien que ceux-ci présentaient tous des TCP à l'école primaire et que l'âge a été contrôlé dans les analyses de régression, cela ne signifie pas que certaines caractéristiques affectent un enfant de six ans de la même façon qu'elles affecteraient un préadolescent de 13 ans. Ainsi, le fait d'étudier un groupe hétérogène sur le plan de l'âge peut avoir contribué à sous-estimer le nombre de caractéristiques permettant de distinguer 
les deux trajectoires. De plus, peut-être que l'étude de caractéristiques plus proximales à la décroissance des TCP, mesurées au temps final de l'étude, aurait été plus concluante. En fait, il est possible que les vulnérabilités présentes au début de l'étude se soient modifiées dans le temps. Par exemple, les pratiques éducatives parentales pourraient s'être améliorées chez les enfants dont les troubles ont décru, mais pas chez ceux dont les troubles ont persisté. Il serait donc intéressant, dans de futures recherches, de poursuivre dans ce sens et de vérifier si l'évolution des caractéristiques individuelles et familiales serait un meilleur prédicteur de la persistance ou de la décroissance des TCP.

L'étude comporte aussi d'autres limites qui peuvent nuancer l'interprétation des résultats, dont la provenance de l'échantillon. À l'entrée dans l'étude, tous les enfants bénéficiaient déjà de services scolaires spécialisés en raison de leurs difficultés comportementales. S'il est justifié de recourir à un échantillon clinique pour faciliter le recrutement d'un nombre suffisant d'enfants, particulièrement de filles, ayant des TCP (Angold, Costello et Erkanli, 1999; Maughan, Rowe, Messer, Goodman et Meltzer, 2004), il est possible que les enfants de cet échantillon présentent plus de vulnérabilités ou des vulnérabilités plus sévères que les enfants d'un échantillon tiré de la population générale (Angold et al., 1999; Goodman et al., 1997; Rutter, 1997; Waschbusch, 2002). Par un effet plafond des mesures, il se peut qu'il soit plus difficile d'identifier des caractéristiques plus spécifiques à la trajectoire persistante dans la présente étude et dans d'autres études utilisant ce type d'échantillon (Hoeve et al., 2008; Pitzer et al., 2010; Raine et al., 2005). En fait, les mesures utilisées pour évaluer les caractéristiques ne sont peut-être pas suffisamment sensibles pour permettre de discriminer différents sous-groupes parmi des enfants qui présentent tous des difficultés importantes. Par contre, cela ne devrait pas remettre en question les différences observées entre les enfants appartenant à l'une ou l'autre trajectoire, d'autant plus que l'intensité des services reçus a été contrôlée dans les analyses. Il faut toutefois éviter de généraliser ces résultats à la population générale.

Quoique le recours à un tel échantillon ait permis de recruter un nombre plus élevé d'enfants - et plus particulièrement de filles qui présentent des TCP en bas âge -, il n'en demeure pas moins que le petit nombre de filles dans la trajectoire persistante pourrait avoir nui à la détection de différences significatives entre les trajectoires et selon le genre. D'ailleurs, plusieurs interactions avec le genre sont marginalement significatives. Un nombre plus élevé de filles 
augmenterait la puissance statistique et permettrait possiblement de détecter des effets de plus petite taille qui s'avèreraient cliniquement utiles (Cohen, 1988). De plus, la persistance et la décroissance des TCP ont été étudiées sur une période de six ans. Il n'est pas rare que ces troubles diminuent chez certains jeunes, mais qu'ils refassent surface ultérieurement (Bushway, Thornberry et Krohn, 2003; Loeber et Stouthamer-Loeber, 1998; Stouthamer-Loeber, Wei, Loeber et Masten, 2004). Il est donc impossible de prédire si la décroissance des TCP observée se maintiendra dans le temps, pour tous les enfants. Des études couvrant une plus grande période de temps, tout en incluant un nombre élevé de filles, permettraient notamment de préciser davantage cette question.

En dépit des limites soulevées, à son terme, cette étude a permis d'approfondir les connaissances sur l'identification des trajectoires des TCP en confirmant les trajectoires obtenues dans les études antérieures et en montrant que ces trajectoires, plus souvent étudiées auprès des garçons, s'appliquaient aussi aux filles. Quant aux caractéristiques individuelles et familiales associées aux différentes trajectoires, l'étude met en évidence que des enfants qui présentent des TCP, peut-être encore davantage les filles, peu importe que ces troubles persistent ou non, font face, initialement, à de nombreuses conditions adverses. Des interventions doivent donc être offertes pour tous ces enfants, même si certains n'adoptent pas une trajectoire persistante de TCP. En fait, ces enfants pourraient développer d'autres difficultés d'adaptation qui n'ont pas été considérées dans la présente étude (Costello, Mustillo, Erkanli, Keeler et Angols, 2003; Diamantopoulou, Verhulst et Van der Ende, 2011; Maughan et al., 2004; Mick et al., 2011; Owens, Hinshaw, Lee et Lahey, 2009; Pardini et Fites, 2010).

Mais encore, les interventions devraient porter non seulement sur les difficultés comportementales, mais aussi cibler les vulnérabilités familiales, dont la santé mentale des parents. Le plus souvent, particulièrement en milieu scolaire, les interventions ciblent les comportements des enfants (Lapalme, Touchette, Déry et Pauzé, 2010). Bien que des interventions visant la diminution des comportements problématiques soient nécessaires, elles ne sont surement pas suffisantes pour changer l'issue développementale des TCP. Selon les résultats de la présente étude et en s'appuyant sur les modèles explicatifs du développement et de la persistance des TCP (Lahey et al., 1999; Lahey et Waldman, 2003; Moffitt, 1993, 2003), lorsque les enfants présentent des 
TCP, un soutien aux parents devrait nécessairement être apporté afin de diminuer les effets délétères des vulnérabilités parentales sur la qualité des interactions parent-enfant et, éventuellement, sur la persistance des TCP et le développement d'autres problèmes d'adaptation.

\section{Références}

Achenbach, T.M. et Edelbrock, C. (1983). Manual for the Child Behavior Checklist and Revised Child Profile. Burlington, VT : Queen City Printers Inc.

American Psychiatric Association (1987). DSM-III-R : Diagnostic and Statistical Manual of Mental Disorders. (3e éd. révisée). Washington, DC : American Psychiatric Publishing.

American Psychiatric Association (2000). DSM-IV-TR : Diagnostic and Statistical Manual of Mental Disorders. (4e éd. révisée). Washington, DC : American Psychiatric Publishing.

American Psychiatric Association (2013).DSM-5 : Diagnostic and Statistical Manual of Mental Disorders, (5 éd.). Washington D.C. : American Psychiatric Association.

Angold, A., Costello, E.J. et Erkanli A. (1999). Comorbidity. Journal of Child Psychology and Psychiatry, 40, 57-87. doi:10.1111/1469-7610.00424

Barker, E.D. et Maughan, B. (2009). Differentiating early-onset persistent versus childhood-limited conduct problem youth. American Journal of Psychiatry, 166, 900-908. doi:org/10.1176/appi.ajp.2009.08121770

Beekhoven, S. et Dekkers, H. (2005). Early school leaving in the lower vocational track : Triangulation of qualitative and quantitative data. Adolescence, 40, 197-213.

Biederman, J., Petty, C. R., Dolan, C., Hughes, S., Mick, E., Monuteaux, M. C. et Faraone, S.V. (2008). The long term longitudinal course of oppositional defiant disorder and conduct disorder in ADHD boys: Findings from a controlled 10-year prospective longitudinal follow-up study. Psychological Medicine, 38, 1027-1036. doi :org/10.1017/S0033291707002668

Boylan, K., Vaillancourt, T., Boyle, M. et Szatmari, P. (2007). Comorbidity of internalizing disorders in children with oppositional defiant disorder. European Child and Adolescent Psychiatry, 16, 484-494. doi:10.1007/ s00787-007-0624-1

Breton, J.J., Bergeron, L., Valla, J.-P., Berthiaume, C. et St-Georges, M. (1998). The Diagnostic Interview Schedule for Children (DISC 2.25) in Quebec, Reliability findings in the light of the MECA study. Journal of American Academy of Child and Adolescent Psychiatry, 37, 1167-1174. doi:org/10.1097/00004583-199811000-00016 
Bushway, S.D., Thornberry, T.P. et Krohn, M.D. (2003). Desistance as a developmental process : A comparison of static and dynamic approaches. Journal of Quantitative Criminology, 19, 129-153. doi: org/10.1023/A:1023050103707

Canino, G., Polanczyk, G., Bauermeister, J. J., Rohde, L. A. et Frick, P. J. (2010). Does the prevalence of CD and ODD vary across cultures? Social Psychiatry and Psychiatric Epidemiology, 45, 695-704. doi:org/10.1007/ s00127-010-0242-y

Cohen, J. (1988). Statistical power analysis for the behavioral sciences (2e éd.). Hillsdale, NJ : Erlbaum.

Cohen, J., Cohen, P., West, S. G. et Aiken, L. S. (2003). Applied multiple regression/correlation analysis for the behavioral sciences ( $3^{\mathrm{e}} \mathrm{ed}$.). Hillsdale, NJ : Erlbaum.

Costello, E. J., Mustillo, S., Erkanli, A. Keeler, G. et Angold, A. (2003). Prevalence and development of psychiatric disorders in childhood and adolescence. Archive of General Psychiatry, 60, 837-844. doi:10.1001/ archpsyc.60.8.837

Costello, J., Egger, H. et Angold, A. (2005). 10-year research update review: The epidemiology of child and adolescent psychiatric disorders: I. Methods and public health burden. Journal of Academy of Child and Adolescent Psychiatry, 44, 972-986. doi:org/10.1097/01.chi.0000172552.41596.6f

Côté, S., Vaillancourt, T., LeBlanc, J. C., Nagin, D. S. et Tremblay, R. E. (2006). The development of physical aggression from toddlerhood to pre-adolescence: A nation wide longitudinal study of Canadian children. Journal of Abnormal Child Psychology, 34(1), 68-82. doi:org/10.1007/s10802-005-9001-z

Déry, M., Toupin, J., Pauzé, R. et Verlaan, P. (2004). Frequency of mental health disorder in a sample of elementary school students receiving special educational services for behavioral difficulties. Canadian Journal of Psychiatry, 49, 769-775. doi :org/10.1177/070674370404901108

Déry, M., Toupin, J., Pauzé, R. et Verlaan, P. (2005). Les caractéristiques d'élèves en difficultés de comportement placés en classe spéciale ou intégrés dans la classe ordinaire. Revue canadienne de l'éducation, 28, 1-23. doi :10.2307/1602151

Diamantopoulou, S., Verhulst, F.C. et van der Ende, J. (2011). The parallel development of ODD and CD symptoms from early childhood to adolescence. European Child and Adolescent Psychiatry, 20, 301-309. doi :org/10.1007/s00787-011-0175-3

Dunn, L.M. et Thériault-Whalen, C.M. et Dunn, L.M. (1993). Échelle de vocabulaire en images Peabody, Toronto : Éditions PSYCan.

Fergusson, D. M., Boden, J. M. et Horwood, L. J. (2009). Situational and generalised conduct problems and later life outcomes: Evidence from a New Zealand birth cohort. Journal of Child Psychology and Psychiatry, 50, 1084-1092. doi: 10.1111/j.1469-7610.2009.02070.x 
Fournier, L., Lesage, A.D., Toupin, J. et Cyr, M. (1997). Telephone surveys as an alternative for estimating prevalence of mental disorders and service utilization : A Montreal catchment area study. Canadian Journal of Psychiatry, 47, 737-742. doi:org/10.1177/070674379704200706

Franzen, M. D., Tishelman, A. C., Sharp, B. H. et Friedman, A. G. (1987). An investigation of the test-retest reliability of the Stroop color-word test across two intervals. Archives of Clinical Neuropsychology, 2, 265-272. doi:org/10.1093/arclin/2.3.265

Frick, P. J. (1991). The Alabama Parenting Questionnaire. University of Alabama.

Goodman, S.H., Lahey, B.B., Fielding, B., Dulcan, M. Narrow, W. et Régier, D. (1997). Representativeness of clinical samples of youths with mental disorders : A preliminary population-based study. Journal of Abnormal Psychology, 106, 3-14. doi:10.1037/0021-843X.106.1.3

Hegvik, R.L., McDevitt, S.C. et Carey, W.B. (1982). The middle childhood temperament questionnaire. Developmental and Behavioral Pediatrics, 3, 197-200. doi:10.1097/00004703-198212000-00004

Hoeve, M., Blokland, A., Dubas, J.S., Loeber, R., Gerris, J.R.M. et van der Laan, P.H. (2008). Trajectories of delinquency and parenting styles. Journal of Abdnormal Child Psychology, 36, 223-235. doi:10.1007/ s10802-007-9172-x

Huesmann, L. R., Eron, L. D., Lefkowitz, M. M. et Walder, L. O. (1984). Stability of aggression over time and generations. Developmental Psychology, 20, 1120-1134. doi:10.1037/0012-1649.20.6.1120

Lahey, B. B., Loeber, R., Burke, J. D. et Applegate, B. (2005). Predicting future antisocial personality disorder in males from a clinical assessment in childhood. Journal of Consulting and Clinical Psychology, 73, 389-399. doi:10.1037/0022-006X.73.3.389

Lahey, B.B, Waldman, I.D. et McBurnett, K. (1999). The development of antisocial behavior : An integrative causal model. Journal of Child Psychology and Psychiatry, 40, 669-682. doi:10.1111/1469-7610.00484

Lahey, B.B et Waldman, I.D. (2003). A developmental propensity model of the origins of conduct problems during childhood and adolescence. In B.B. Lahey, T.E., Moffitt et A. Caspi (dir.), Causes of conduct disorder and juvenile delinquency (pp.76-117). New York : Guilford Press.

Lapalme, M., Touchette, L., Déry, M. et Pauzé, R. (2010). Mesure de l'efficacité des services reçus par les parents d'enfants desservis par les Centres jeunesse du Québec. Dans M. Déry, A.-S. Denault et J.-P. Lemelin (dir.), Aide aux jeunes en difficulté de comportement : regard sur nos pratiques (pp. 49-69). Sherbrooke, Qc : Groupe de recherche sur les inadaptations sociales de l'enfance, Université de Sherbrooke.

Lezak, M. D., Howieson, D. B. et Loring, D. W. (2004). Neuropsychological Assessment (4e éd.). New York : Oxford University Press. 
Loeber, R. et Keenan, K. (1994). The interaction between conduct disorder and its comorbid conditions: effects of age and gender. Clinical Psychology Review, 14, 497-523. doi:org/10.1016/0272-7358(94)90015-9

Loeber, R. et Stouthamer-Loeber, M. (1998). Development of juvenile aggression and violence : Some common misconceptions and controversies. American Psychologist, 53, 242-259. doi :10.1037/0003066X.53.2.242

Lussier, F. et Flessas, J. (2009). Neuropsychologie de l'enfant : troubles développementaux et de l'apprentissage ( $2^{\mathrm{e}}$ éd.). Paris, France : Dunod.

Maughan, B., Rowe, R., Messer, J., Goodman, R. et Meltzer, H. (2004). Conduct disorder and oppositional defiant disorder in a national sample : developmental epidemiology. Journal of Child Psychology and Psychiatry, 45, 609-621. doi: 10.1111/j.1469-7610.2004.00250.x

Meltzer, H., Gatwood, R., Goodman, R. et Ford, T. (2000). Mental health of children and adolescents in Great Britain. TSO : London.

McLachlan, G. et Peel, D. (2000). Finite mixture models. New York : Wiley.

Mick, E., Byrne, D., Fried, R., Monuteaux, M., Faraone, S. V. et Biederman, J. (2011). Predictors of ADHD persistence in girls at 5-year follow-up. Journal of Attention Disorders, 15, 183-192. doi:o $\mathrm{rg} / 10.1177 / 1087054710362217$

Moffitt, T. E. (1993). Adolescence-limited and life-course-persistent antisocial behavior : A developmental taxonomy. Psychological Review, 100, 674-701. doi:10.1037/0033-295X.100.4.674

Moffitt,T.E.(2003). Life-course persistent and adolescence-limited antisocial behavior. In B.B. Lahey, T.E. Moffitt et A. Caspi (Eds.), Causes of conduct disorder and juvenile delinquency (pp. 49-75). New York: Guilford.

Moffitt, T. E. (2006). Life-course-persistent and adolescent-limited antisocial behavior. In D. Cicchetti et D. J. Cohen (Eds.), Developmental psychopathology : Vol. 3. Risk, disorder and adaptation (pp. 570-598). New York : Wiley. doi:10.1002/9780470939406.ch15

Moffitt, T.E., Caspi, A., Dickson, N., Silva, P. et Stanton, W. (1996). Childhood-onset versus adolescent-onset antisocial conduct problems in males : Natural history form ages 3 to 18 years. Development and Psychopathology, 8, 399-424. doi:org/10.1017/S0954579400007161

Moffitt, T.E., Caspi, A., Harrington, H. et Milne, B.J. (2002). Males on the life-course persistent and adolescence-limited antisocial pathways: Follow-up at age 26 years. Development and Psychopathology, 14, 179207. doi :10.1017/S0954579402001104

Muthén, L.K. et Muthén, B.O. (1998-2008). Mplus User's Guide (4éd.). Los Angeles, CA : Muthén et Muthén.

Owens, E. B., Hinshaw, S. P., Lee, S. S. et Lahey, B. B. (2009). Few girls with childhood attention-deficit/hyperactivity disorder show positive adjustment during adolescence. Journal of Clinical Child \& Adolescent Psychology, 38, 132-143. doi:org/10.1080/15374410802575313 
Pardini, D. A. et Fite, P. J. (2010). Symptoms of conduct disorder, oppositional defiant disorder, attention-deficit/hyperactivity disorder, and callousunemotional traits as unique predictors of psychosocial maladjustment in boys: Advancing an evidence base for DSM-V. Journal of the American Academy of Child and Adolescent Psychiatry, 49, 1134-1144. doi:org/10.1016/j.jaac.2010.07.010

Piquero, A. R., Farrington, D. P., Nagin, D. S. et Moffitt, T. E. (2010). Trajectories of offending and their relation to life failure in late middle age: Findings from the Cambridge Study in Delinquent Development. Journal of Research in Crime and Delinquency, 47(2), 151-173. doi:org/10.1177/0022427809357713

Pitzer, M., Schmidt, M.H. et Laucht, M. (2010). Early predictors of antisocial developmental pathways among boys and girls. Acta Psychiatrica Scandinavica, 121, 52-64. doi:10.1111/j.1600-0447.2009.01411.x

Raine, A., Moffitt, T.E., Caspi, A., Loeber, R., Stouthamer-Loeber, M. et Lynam, D. (2005). Neurocognitive impairments in boys on the life-course persistent antisocial path. Journal of Abnormal Psychology, 114, 38-49. doi:10.1037/0021-843X.114.1.38

Raftery, A.E. (1995). Bayesian model selection in social research. Social Methodology, 25, 111-164. doi:10.2307/271063

Renk, K. (2008). Disorders of conduct in young children: Developmental considerations, diagnoses, and other characteristics. Developmental Review, 28, 316-341. doi:org/10.1016/j.dr.2007.01.001

Rey, A. (1959). Manuel du Test de copie et de reproduction de mémoire de figures géométriques complexes. Paris : Les Éditions du Centre de Psychologie Appliquée.

Robins, L. N., Helzer, J. E., Orvaschel, H., Anthony, J. C., Blazer, D. G., Burnam, A. et Burke, J. D., JR. (1985). In W. W. Eaton et L. G. Kessler (Eds.), Epidemiological field methods in psychiatry: NIMH Epidemiological Catchment Area program (chap. 8). New York: Academic Press.

Rowe, R., Costello, E.J., Angold, A., Copeland, W.E. et Maughan, B. (2010). Developmental pathways in oppositionnal defiant disorder and conduct disorder. Journal of Abnormal Psychology, 119, 726-738. doi:10.1037/ a0020798

Rutter, M. (1997). Comorbidity : Concepts, claims and choices. Criminal Behaviour and Mental Health, 7, 265-285. doi: 10.1002/cbm.190

Serbin, L. A., Cooperman, J. M., Peters, P. L., Lehoux, P. M., Stack, D. M. et Schwartzman, A. E. (1998). Inter-generational transfer of psychosocial risk in women with childhood histories of aggression, withdrawal, or aggression and withdrawal. Developmental Psychology. 34:1246-62. doi:10.1037/0012-1649.34.6.1246 
Shaffer, D., Schwab-Stone, M., Fisher, P., Cohen, P., Piacentini, J., Davies, M., Conners, C.K. et Regier, D. (1993). Diagnostic Interview Schedule for Children-Revised (DISC-R) : I. Preparation, field testing, interrater reliability and acceptability. Journal of the American Academy of Child and Adolescent Psychiatry, 32, 643-650. doi: org/10.1097/00004583199305000-00023

Shelton, K.K., Frick, P.J. et Wooton, J. (1996). Assessment of parenting practices in families of elementary school-age children, Journal of Clinical Child Psychology, 25, 317-329. doi:org/10.1207/ s15374424jccp2503_8

Snyder, J. et Patterson, G. (1987). Family interaction and delinquent behavior. In H. Quay (Ed.), Handbook of juvenile delinquency (pp. 216243). New York: Wiley.

SPSS Inc. (2008). SPSS Statistics for Windows, Version 17.0. Chicago : SPSS Inc.

Stouthamer-Loeber, M., Wei, E., Loeber, R. et Masten, A.S. (2004). Desistance from persistent serious delinquency in the transition to adulthood. Development and Psychopathology, 16, 897-918. doi: org/10.1017/ S0954579404040064

Valla, J.-P., Bergeron, L., Bérubé, H., Gaudet, N. et St-Georges, M. (1994). A structured pictorial questionnaire to assess DSM-III-R-based diagnoses in children (6-11 years): development, validity and reliability. Journal of Abnormal Child Psychology, 22, 403-423. doi: org/10.1007/BF02168082

Van Domburgh, L., Loeber, R., Bezemer, D., Stallings, R. et StouthamerLoeber, M. (2009). Childhood predictors of desistance and level of persistence in offending in early onset offenders. Journal of Abnormal Child Psychology, 37, 967-980. doi:org/10.1007/s10802-009-9329-x

Veenstra, R., Lindenberg, S., Verhulst, F.C. et Ormel, J. (2009). Childhoodlimited versus persistent antisocial behavior : Why do some recover and others do not? The TRAILS study. The Journal of Early Adolescence, 29, 718-742. doi:org/10.1177/0272431608325501

Waschbusch, D. A. (2002). A meta-analytic examination of comorbid hyperactive-impulsive-attention problems and conduct problems. Psychological Bulletin, 128, 118-150. doi:10.1037/0033-2909.128.1.118

Whittinger, N., Langley, K., Fowler, T., Thomas, H. et Thapar, A. (2007). Clinical precursors of adolescent conduct disorder in children with attention-deficit hyperactivity disorder. Journal of the American Academy of Child and Adolescent Psychiatry, 46, 179-187. doi:org/10.1097/01.chi.0000246066.00825.53

Zahn-Waxler, C. et Polanichka, N. (2004). All things interpersonal: Socialization and female aggression. In M. Putallaz et K. Bierman (Eds), Aggression, antisocial behavior and violence among girls: A developmental perspective (pp. 48-68). New York: Guilford. 


\section{Correspondance :}

\section{Mélanie Lapalme}

Département de psychoéducation

Université de Sherbrooke

2500, boulevard de l'Université

Sherbrooke, Québec

Canada, J1K 2R1

Tél. : +1 819 821-8000, poste 62565

Courriel : melanie.lapalme@usherbrooke.ca

\section{Résumé}

Caractéristiques individuelles et familiales associées à la persistance des troubles du comportement perturbateur chez les enfants. Cette étude vise à identifier des caractéristiques distinguant les enfants des trajectoires persistante et décroissante des troubles de comportement perturbateur et de vérifier l'effet modérateur du genre. L'échantillon est composé de 268 enfants ( $28 \%$ de filles) du primaire ( $M=9,8$ ans; é- $t .=1,79$ an) recevant des services spécialisés pour difficultés comportementales à l'école. Selon les analyses de trajectoires (six ans) réalisées, 12,3\% d'enfants (24\% de filles) appartiennent à la trajectoire persistante et $32,5 \%$ (30\% de filles) à la trajectoire décroissante. Bien que les enfants des deux trajectoires présentent des vulnérabilités similaires à l'entrée dans l'étude, les analyses de régression multivariées indiquent qu'un tempérament plus réactif augmente la probabilité d'appartenir à la trajectoire persistante chez les filles. Aussi, la présence d'un trouble anxieux et l'absence d'un trouble dépressif chez les parents seraient associées à la trajectoire persistante. Ces résultats soutiennent l'importance de cibler aussi les difficultés des parents lorsque l'on souhaite prévenir la persistance des troubles du comportement.

Mots-clés : troubles du comportement; trajectoires de développement; caractéristiques individuelles et familiales; enfance; effets de genre. 


\section{Abstract}

Individual and family characteristics associated with the persistence of disruptive behavior disorders in children. This study identifies characteristics, measured at study entry, that differentiate between children engaging in persisting or in decreasing trajectories of behavior disorder and verifies the moderating effect of gender. The sample consists of 268 elementary school-age children (28\% of girls; $\mathrm{M}=9.8$ years, s.d.=1.79) receiving services for behavioral problems at school. According to the latent growth class analysis (six years), $12.3 \%$ of children (24\% of females) belonged to the persisting trajectory and $32.5 \%$ (30\% of female) belonged to the descending trajectory. Although children in both trajectories had similar vulnerabilities at study entry, multivariate regression analyzes identified some differentiating characteristics: a temperament characterized by a high sensory threshold increases the probability of belonging to the persistent trajectory among girls; the presence of an anxiety disorder and the absence of a depressive disorder in the parents was associated with membership in the persistent trajectory. These findings support targeting difficulties of parents to prevent the persistence of behavioral problems.

Keywords: behavior disorders; trajectory; individual and familial factors; childhood; gender. 